\title{
Evidence for a Nuclear Electric Quadrupole Moment for $\mathrm{Sb}^{123}$
}

\author{
D. H. Tomboulian and R. F. Bacher \\ Cornell University, Ithaca, New York
}

(Received May 11, 1940)

\begin{abstract}
Measurements of the hyperfine structure of the line $\lambda 5895$ of Sb II by the use of a Fabry-Perot interferometer have shown that there are deviations from the interval rule in the case of the isotope with mass number 123 . The hyperfine structure intervals of the ${ }^{3} D_{1}$ level of $\mathrm{Sb}^{121}(\mathrm{I}=5 / 2)$ are found to be 0.6801 and $0.4832 \mathrm{~cm}^{-1}$. The corresponding intervals in $\mathrm{Sb}^{123}(\mathrm{I}=7 / 2)$ are 0.4814 and $0.3603 \mathrm{~cm}^{-1}$. The error in these measurements is of the order of $0.001 \mathrm{~cm}$. The observed ratio of the two separa-
\end{abstract}

tions gives 1.407 and 1.336 for the light and heavy isotope, respectively, whereas the corresponding ratios on the basis of the interval rule are 1.400 and 1.286 . The deviation in the case of the light isotope is within the experimental error. The deviation for the heavy isotope, however, is a real effect which cannot be accounted for on the basis of perturbing effects of neighboring levels and must therefore be ascribed to the presence of an electric quadrupole moment of the nucleus of $\mathrm{Sb}^{123}$.
$I^{\mathrm{N}}$ NVESTIGATIONS ${ }^{1,2}$ of the hyperfine structure in the spectrum of Sb II have revealed patterns that are extremely complex. Because of the comparable relative abundance of the two isotopes (mass number 121, and 123) and because most of the intense lines involve transitions between levels of high $J$ value, the observed patterns suffer from serious blending. In the spectral range $5000-7000 \mathrm{~A}$, the line $\lambda 5895$ is among the few which show sufficiently simple and well resolved structure. This line has been assigned to the transition $5 p 6 p^{3} D_{1} \rightarrow 5 p 6 s^{3} P_{0}$ by Krishnamurty. ${ }^{3}$ Because of the complexity of the atomic system and particularly because of the large number of unassigned levels nearby, this assignment cannot be considered as certain. However, there is no question from the hyperfine structure of the line which shows six resolved components, that the line is a transition from a state $J=1$ to a state $J=0$. Since this is sufficient information to make a comparison of the nuclear effects produced by the two isotopes, the present investigation was undertaken for the purpose of making accurate measurements on the structure of this line.

The spectrum was excited in a hot hollow cathode tube through which purified helium was circulated. In operating the tube considerable difficulty was encountered in arriving at conditions favorable for the production of a strong, sharp spectrum. Tube currents of 100 to $200 \mathrm{ma}$ were used; the pressure of the helium gas was

\footnotetext{
${ }^{1}$ J. S. Badami, Zeits. f. Physik 79, 206 (1932).

${ }_{2}$ S. Tolanski, Proc. Roy. Soc. A146, 182 (1934)

${ }^{3}$ S. G. Krishnamurty, Ind. J. Phys. 10, 90 (1937).
}

kept as low as possible for the maintenance of a steady discharge. The sample of antimony was free from the oxide and other impurities were less than 0.03 percent. The high resolution apparatus consisted of a Fabry-Perot interferometer which was mounted in the collimated beam of a Zeiss three-prism spectrograph. A series of separators, in the 2- to 5-mm range, was employed in conjunction with heavily silvered plates. Exposure times were of the order of several minutes.

The energy level diagram and the observed line structure for $\lambda 5895$ is shown in Fig. 1. The solid and dotted lines refer, respectively, to the light and heavy isotopes. The measurements on the various intervals are summarized in Table I. In the reduction of the plates 8 to 10 orders of interference were included. The largest deviation from the mean in the measurement of any one interval is $0.0019 \mathrm{~cm}^{-1}$. The present results are considered more accurate than those given by previous measurements, ${ }^{1,2}$ in which in tervals $1-2$, $2-3$ and $3-4$ show wide variations. In particular, Tolanski ${ }^{2}$ states that the deviation in the separation of the smallest interval is $0.019 \mathrm{~cm}^{-1}$. In the present data the deviation in the same interval (3-4) is of the order of $0.001 \mathrm{~cm}^{-1}$.

The results obtained by Crawford and Bateson $^{4}$ from a study of Sb IV show that the nuclear spin of $\mathrm{Sb}^{121}$ is $5 / 2$ and that of $\mathrm{Sb}^{123}$ is $7 / 2$. Their conclusions were based upon a study of the line $\lambda 3537 \mathrm{~A}\left(5 s 6 p^{3} P_{0} \rightarrow 5 s 6 s^{3} S_{1}\right)$ and are in agreement with the previous conclusions of Badami. ${ }^{1}$ This

\footnotetext{
${ }^{4}$ M. F. Crawford and S. Bateson, Can. J. Research 10, 693 (1934).
} 
line, which they completely resolved, is free from quadrupole effects so that the nuclear spins can be determined directly from an application of the interval rule. From their values of the nuclear spins, it is possible to compare the separations of the $5 p 6 p^{3} D_{1}$ level of Sb II with those to be expected on the basis of the interval rule (see Table II).

Since there usually is a likelihood of underestimating the separation of close components it is of importance to note the spread of the observed interval ratios. From the data of Table I it is found that for $\mathrm{Sb}^{121}$, the largest and smallest values of this ratio are 1.415 and 1.395 , respectively. Similar quantities for $\mathrm{Sb}^{123}$ are 1.344 and 1.323. For $\mathrm{Sb}^{121}$, the observed deviation from the interval rule is probably within the experimental error, but for $\mathrm{Sb}^{123}$ the observed deviation is decidedly outside the inaccuracies of measurement and therefore is a real effect.

Deviations from the interval rule have been successfully ascribed in general either to the presence of a nuclear electric quadrupole moment or to the effect of perturbations of nearby terms. In the present case, the $\mathrm{Sb}^{121}$ separations obey the interval rule almost exactly while for those of $\mathrm{Sb}^{123}$, the observed ratio shows a deviation of 3.9 percent. This large difference for the two isotopes indicates that the effect cannot be ascribed to perturbations by nearby levels. Furthermore, the observed deviation for $\mathrm{Sb}^{123}$ is quite large and would be very difficult to account for even without the above argument. On the

TABLE I. Hyperfine structure intervals $\lambda 5895 \mathrm{Sb}$ II.

\begin{tabular}{c|ccccc}
\hline \hline $\begin{array}{c}\text { SEPARATOR } \\
\text { (MM) }\end{array}$ & $1-2$ & $2-3$ & $3-4$ & $4-5$ & $5-6$ \\
\hline 2.780 & 113.3 & 482.2 & 84.8 & 274.5 & 208.1 \\
3.691 & 112.7 & 482.0 & 85.7 & 274.2 & 208.2 \\
3.691 & 113.2 & 481.9 & 85.6 & 274.0 & 208.5 \\
4.054 & 114.2 & 479.7 & 85.4 & 276.8 & 208.6 \\
\hline Averages & 113.3 & 481.4 & 85.4 & 274.9 & 208.3 \\
\hline \hline
\end{tabular}

TABLE II. Separations of ${ }^{3} D_{1}$ level.

\begin{tabular}{ccccc}
\hline Isotope & $\begin{array}{c}\text { SePaRATION } \\
\text { OF }{ }^{3} D_{1}\end{array}$ & $\begin{array}{c}\text { OBSERVED } \\
\text { RATIO }\end{array}$ & $\begin{array}{c}\text { RATIO FROM } \\
\text { INTERAL } \\
\text { RULE }\end{array}$ \\
\hline 121 & 680.1 & 483.2 & 1.407 & 1.400 \\
123 & 481.4 & 360.3 & 1.336 & 1.286 \\
\hline \hline
\end{tabular}

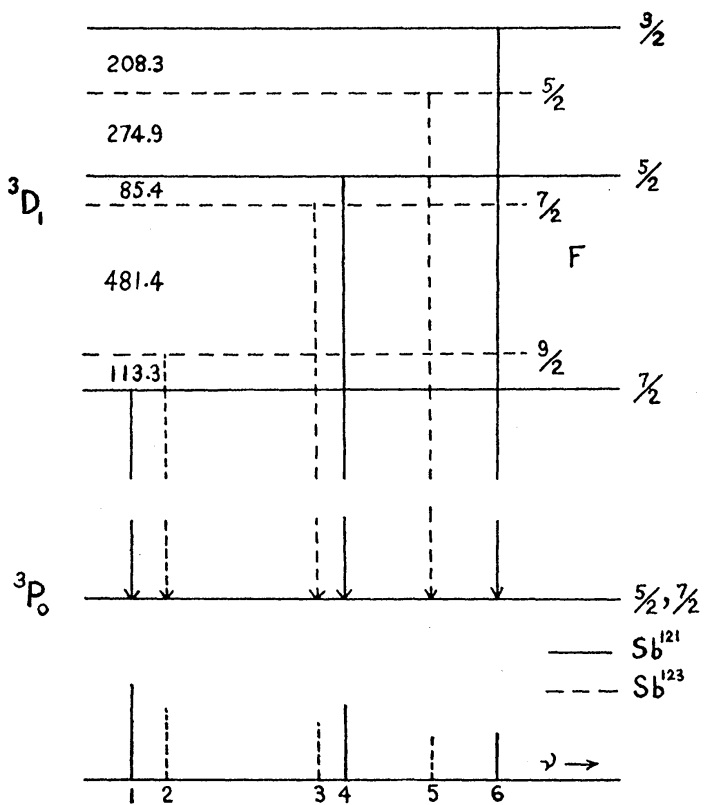

FIG. 1. Level diagram and line structure $\lambda 5895$ of Sb II.

basis of observed levels the nearest neighbor is $86 \mathrm{~cm}^{-1}$ above. It has $J=2$ and has been ascribed to $5 p 6 p^{3} D_{2}$ and the order of its perturbing effect can be obtained from the ratio $A^{2} / d$, where $A$ is the h.f.s. separation factor and $d$ the distance to the nearest level. For $\mathrm{Sb}^{123} A=0.105 \mathrm{~cm}^{-1}$, the effect of a level $86 \mathrm{~cm}^{-1}$ away would be of the order of $10^{-4} \mathrm{~cm}^{-1}$ which is negligible. It is, of course, possible that a state as yet undiscovered lies nearer than the one we have considered. Under these circumstances the relative perturbations for the two isotopes would be given by

$$
\frac{P_{121}}{P_{123}}=\frac{\left(A_{121}\right)^{2}}{\left(A_{123}\right)^{2}}=\left(\frac{96.9}{52.6}\right)^{2}=3.4 \text {. }
$$

A larger perturbation would be expected for $\mathrm{Sb}^{121}$. This is the isotope for which the interval ratio is found to agree with the expected value. From the above considerations it appears that the observed deviation from the interval rule for $\mathrm{Sb}^{123}$ must be ascribed to some other cause and presumably to the presence of an electric quadrupole moment.

The atomic state which gives this quadrupole effect has been assigned to $5 p 6 p^{3} D_{1}$. This assignment is somewhat uncertain due to the intermediate coupling between the two electrons and 


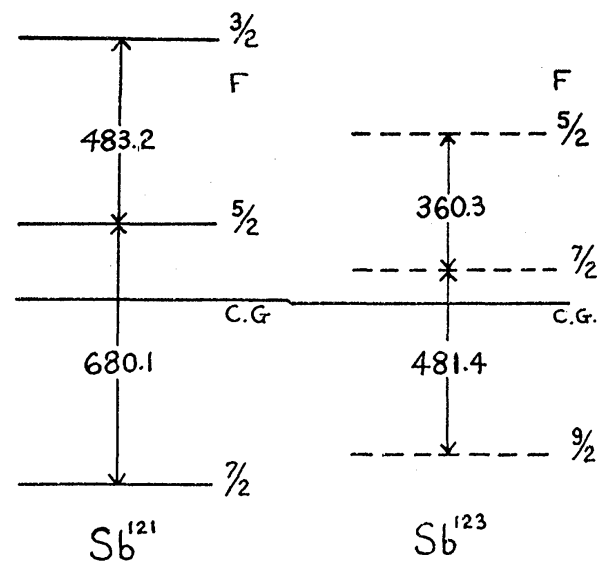

FIG. 2. Observed separations of ${ }^{3} D_{1}$ level.

possibly also due to perturbing effects from the $5 p 4 f$ configuration. While the quadrupole effect is definitely recognizable, it does not seem feasible to attempt a determination of the quadrupole moment from the observed deviations.

As a check on the measurements reported here the ratios of the magnetic moments for the two isotopes can be determined from the total separations of the hyperfine structures. This gives $\mu_{121} / \mu_{123}=1.316$ and is in close agreement with the value 1.32 which is obtained from the separations of the $5 s 6 s^{3} S_{1}$ level of Sb IV studied by Crawford and Bateson. ${ }^{4}$

Finally it is of interest to see whether the observed line shows any isotope shift. This can be done by determining the center of gravity of the observed structures taking the statistical weight of each hyperfine state $(2 F+1)$. As shown in Fig. 2 the centers of gravity for $\mathrm{Sb}^{121}$ and $\mathrm{Sb}^{123}$ are almost coincident. The c.g. for $\mathrm{Sb}^{121}$ lies $0.001 \mathrm{~cm}^{-1}$ above that for $\mathrm{Sb}^{123}$ indicating that the isotope shift in this transition is negligible.

\title{
Note on the Effect of Pressure on the Curie Point of Iron-Nickel Alloys
}

\author{
J. C. Slater \\ Massachusetts Institute of Technology, Cambridge, Massachusetts
}

(Received April 25, 1940)

By applying Clapeyron's equation, an estimate is made of the increase of the Curie point of nickel with pressure, which comes out of the order of magnitude of $5 \times 10^{-5}$ degree per atmosphere. For iron-nickel alloys, the increase of Curie point with pressure would become less as more iron is added, becoming zero with something like 70 percent of nickel, and for the alloys containing more iron the Curie

$I^{\mathrm{T}}$ is a matter of considerable geophysical interest to know the effect of pressure on the Curie point of iron-nickel alloys such as may constitute the core of the earth. It is ordinarily supposed that the permanent magnetism of the earth cannot be explained as being of ferromagnetic origin, because at the high temperatures assumed in the interior, of the order of several thousand degrees absolute, the alloys would be far above their Curie points, and would not be ferromagnetic. On the other hand, there are presumably very high pressures, of the order of $10^{6}$ atmospheres, in the interior of the earth, and point would decrease with pressure. These magnitudes are such that it seems most unlikely that pressures existing in the iron-nickel core of the earth would raise the Curie point of the material enough so that it could be ferromagnetic at the temperatures existing inside the earth. This makes any ferromagnetic explanation of the magnetism of the earth most implausible.

if such a pressure should raise the Curie point enough, the interior of the earth might still be ferromagnetic. It thus becomes important to know whether pressure would be expected to raise or to lower the Curie point, and if it raises it, to know whether it could be by some such amount as $5000 / 10^{6}=5 \times 10^{-3}$ degree per atmosphere (taking the temperature to be $5000^{\circ}$ ), which would suffice to make the interior of the earth ferromagnetic. Not many experiments are available to settle this point, on account of the difficulty of combining high pressure and high temperature technique. It becomes important 